\section{Posible asociación autoinmune entre síndrome de Laugier-Hunziker y síndrome de Sjögren: reporte de un caso y revisión de la literatura}

\author{
XIMENA FAJRE ${ }^{1}$, MARÍA ASPILLAGA ${ }^{2}$, MARÍA MCNAB ${ }^{3}$, \\ JORGE NAVARRETE ${ }^{3}$, VERÓNICA SANHUEZA ${ }^{4}$, JUANA BENEDETTO
}

\section{Laugier-Hunziker syndrome in a patient with Sjögren's syndrome. Report of one case}

Laugier-Hunziker syndrome is a rare benign idiopathic condition characterized by acquired macular pigmentation of lips and buccal mucosa, often accompanied with melanonychia. The main concern with this condition is to rule out other differential diagnosis with systemic repercussions and similar hyperpigmentation patterns, such as Peutz-Jeghers syndrome, adrenal insufficiency and melanoma. We report a 58-year-old female with a 20-year history of Sjögren's syndrome, presenting with melanonychia and hyperpigmentation in the buccal mucosa. She had no relevant medication history and is a non-smoker. The patient denied any other symptoms. The histopathology confirmed the diagnosis of Laugier-Hunziker syndrome.

(Rev Med Chile 2016; 144: 671-674)

Key words: Autoimmunity; Hyperpigmentation; Mouth Mucosa; Pigmentation Disorders; Sjogren's syndrome.
'Departamento de Dermatología,

Clínica Alemana de Santiago,

Facultad de Medicina Clínica

Alemana, Universidad del

Desarrollo, Hospital Padre

Hurtado. Santiago, Chile.

${ }^{2}$ Departamento de Dermatología,

Clínica Alemana de Santiago,

Facultad de Medicina Clínica

Alemana, Universidad del

Desarrollo. Santiago, Chile.

${ }^{3}$ Práctica privada. Santiago, Chile.

${ }^{4}$ Departamento de Anatomía

Patológica, Hospital Padre

Hurtado. Santiago, Chile.

Fuente de apoyo financiero:

Ninguna que declarar

Recibido el 4 de agosto de 2015, aceptado el 22 de enero de

2016

Correspondencia a:

Jorge Navarrete

Práctica privada, Luis Carrera

1096, departamento 93,

Vitacura. Santiago, Chile.

http://orcid.org/0000-0002-

8563-0364

jnavarrete90@gmail.com
1 líndrome de Laugier-Hunziker (SLH) es una condición infrecuente, idiopática y benigna, sin manifestaciones sistémicas conocidas. Constituye un diagnóstico de descarte, sin criterios diagnósticos formales (Tabla 1).

La histopatología no es patognomónica, pero junto a la clínica apoya el diagnóstico. Generalmente muestra un incremento significativo de melanina en epidermis, melanocitos de morfología normal en membrana basal (los cuales pueden estar aumentados en cantidad) e incontinencia pigmentaria en dermis papilar, con melanófagos $^{5-7}$. El principal objetivo de una biopsia en el SLH con lesiones focales es descartar melanoma.

Este caso corresponde al primer registro de SLH asociado a una enfermedad del tejido conectivo en Chile ${ }^{8,9}$. Revisando la literatura internacional disponible, existen al menos cinco publicaciones que relacionan SLH a autoinmunidad ${ }^{10-14}$. Lo an- terior nos permite sospechar que un mecanismo autoinmune podría participar en la patogénesis de esta entidad y, por ende, requiere formular un enfrentamiento inicial acorde.

\section{Caso clínico}

Paciente de 58 años, chilena, que consulta a nuestro servicio con historia de 20 años de evolución de síndrome de Sjögren (Tabla 2), tratado únicamente con lágrimas artificiales. Tiene un hijo con vitíligo y una hermana con síndrome de Sjögren. Sin consumo de medicamentos o tabaquismo. Sin antecedentes familiares de cáncer digestivo. Niega síntomas de hemorragia digestiva, dolor abdominal, diarrea, fatiga o baja de peso. Fue derivada desde Reumatología al Servicio de Dermatología del Hospital Padre Hurtado debido 
a la aparición de bandas hiperpigmentadas en uñas de los pies desde hacía 5 años, seguidas por las uñas de las manos. Hacía 3 años que habían aparecido máculas color café en mucosa oral, asociado a hiperpigmentación periungueal progresiva.

\section{Tabla 1. Diagnóstico de síndrome de Laugier-Hunziker}

\section{Hiperpigmentación macular adquirida con las} siguientes características s,4,12 $^{3,}$

- Café, café-azulado a café-negro

- Bien delimitadas o de bordes moderadamente difusos

- Solitarias o confluentes

- Usualmente menores a $5 \mathrm{~mm}$ de diámetro

- Generalmente de inicio en tercera a quinta década de vida

- Persistentes o progresivas

- Leve predominancia en mujeres

\section{Hallazgos frecuentes}

- Máculas en labios, vestíbulo oral y paladar duro

- Hiperpigmentación ungueal (melanoniquia) en 60\%, más frecuente en manos, sin onicodistrofia. Usualmente en forma de bandas longitudinales, únicas o múltiples ${ }^{1,17}$

\section{Hallazgos infrecuentes}

- Máculas café en paladar blando, lengua, encías, comisuras labiales, pulpejos de los dedos y plantas

- Melanoniquia de la totalidad de una o más uñas

\section{Hallazgos raros}

- Hiperpigmentación en genitales, periné, tórax, porción proximal de extremidades, perioral, escleras y esófago 1,2,17

El diagnóstico requiere descartar otras causas, principalmente síndrome de Peutz-Jeghers, insuficiencia suprarrenal y fármacos (ver texto)

Histopatología no característica (ver texto)

Tabla 2. Criterios de clasificación de síndrome de Sjögren ACR 2012

El paciente debe presentar al menos 2 de los 3 siguientes criterios:

1 Anticuerpos anti-Ro y/o anti-La positivos o factor reumatoideo positivo y anticuerpos antinucleares en títulos $\geq 1: 320$

2 Score de tinción ocular $\geq 3$ (en paciente que no esté en tratamiento con lágrimas artificiales)

3 Presencia de sialoadenitis linfocítica focal con un score de focus $\geq 1$ focus $/ 4 \mathrm{~mm}^{2}$ en biopsia de glándula salival labial

*ACR: American College of Rheumatology.
Al examen físico, la mucosa oral presentaba una mácula café en labio inferior hacia comisura labial izquierda [Figura 1 (a)]. En uñas de manos y pies se observaban máculas café periungueales y melanoniquia [Figura 1 (b) y (c)].

La histopatología de mucosa oral mostró acantosis leve e hipermelanosis. En la unión dermoepidérmica se observaron numerosos melanófagos, con capilares prominentes en dermis (Figura 2).

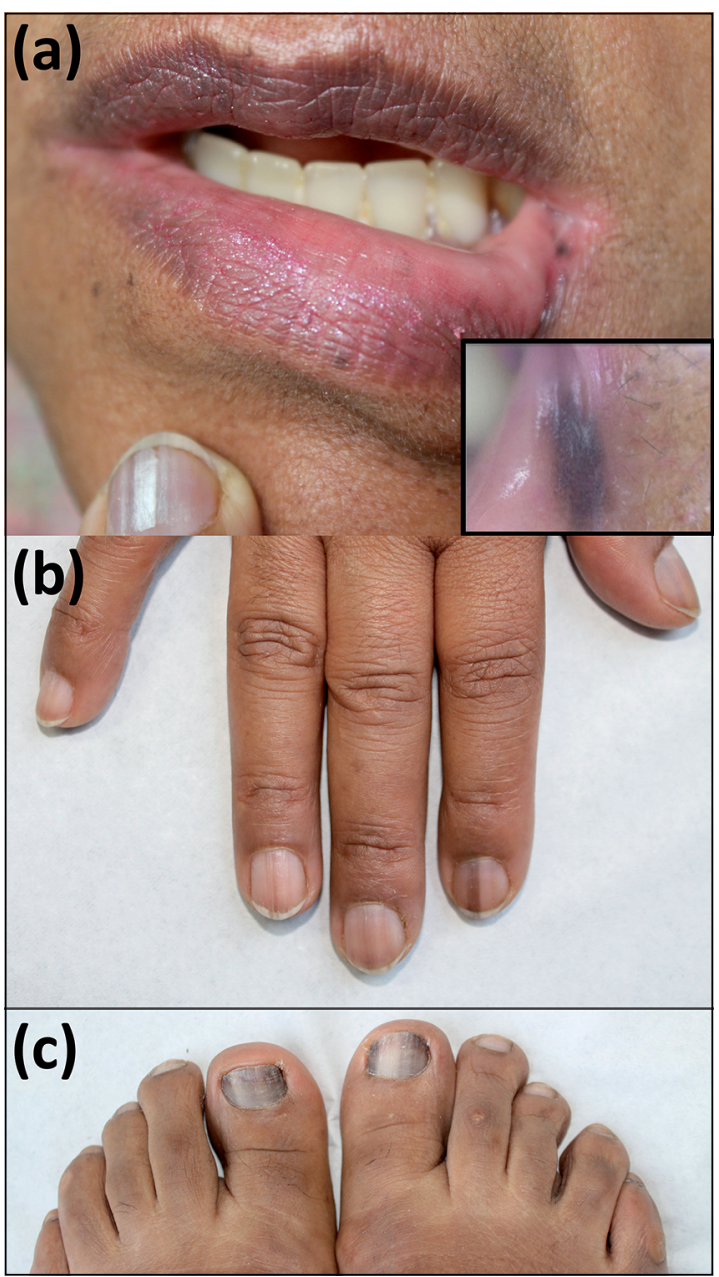

Figura 1. (a) Mácula café en la comisura labial izquierda luego de la biopsia. Recuadro muestra la misma lesión de 4 x $3 \mathrm{~mm}$ de diámetro previa biopsia; (b) Múltiples bandas café en uñas de mano derecha; (c) Lesión hiperpigmentada difusa en uña del primer dedo pie izquierdo y dos bandas longitudinales café de $4 \mathrm{~mm}$ de ancho sobre uña del primer dedo pie derecho. 


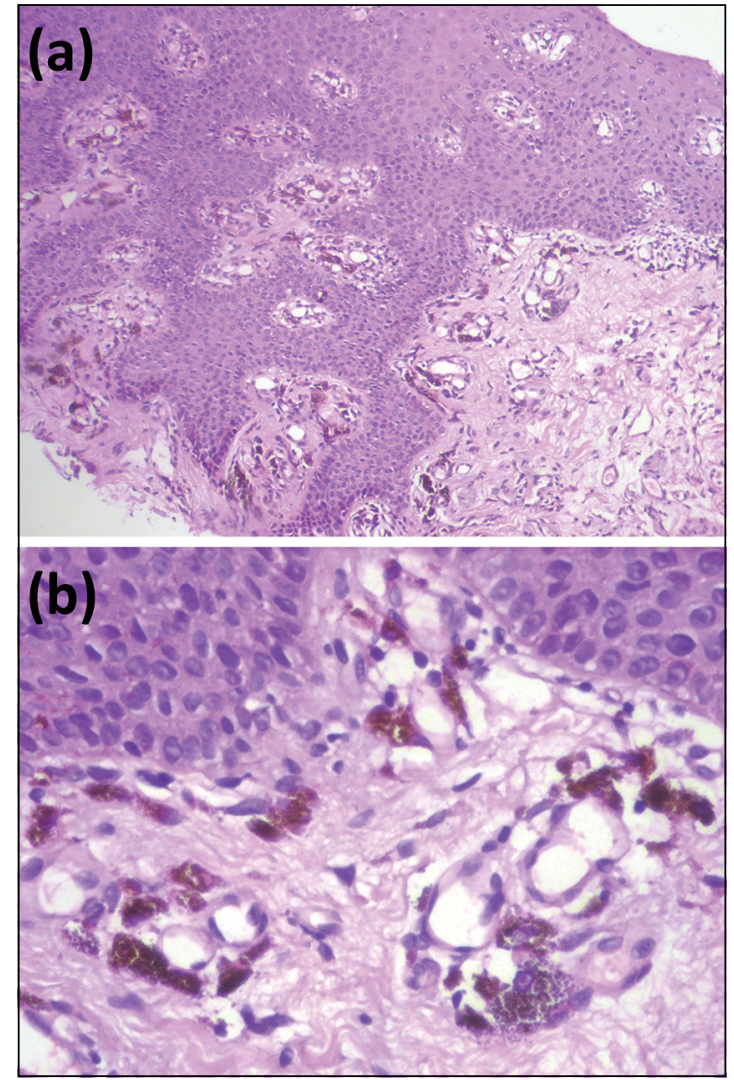

Figura 2. Tinción con hematoxilina-eosina de biopsia mucosa oral. (a) 100x, epidermis con acantosis y melanófagos en dermis papilar; (b) 400x, melanófagos rodeando capilares en dermis papilar.

Los exámenes de laboratorio mostraron: Leucopenia leve $(3,700 / \mathrm{uL}$ con $1,370 / \mathrm{uL}$ linfocitos). El resto del hemograma, velocidad de eritrosedimentasión, hormonas tiroideas, cortisol plasmático y ACTH se encontraban dentro del rango normal.

Los anticuerpos anti-Ro $(43,7 \mathrm{U} / \mathrm{mL})$ y anti-La ( $>48 \mathrm{U} / \mathrm{mL}$ ) fueron positivos, así como también los anticuerpos antinucleares (1/2560) con patrón moteado. Los anticuerpos anti-dsDNA, anti-Sm $\mathrm{y}$ anticardiolipinas fueron negativos. El factor reumatoideo, anticoagulante lúpico y la cuantificación de inmunoglobulinas resultaron dentro de rangos normales. Se realizó endoscopía digestiva alta y colonoscopía, que no muestran presencia de pólipos, hiperpigmentación u otros hallazgos significativos.

La paciente continúa en seguimiento clínico, sin aparición de nuevas lesiones.

\section{Discusión}

El principal objetivo ante la sospecha de SLH consiste en descartar causas de hiperpigmentación mucocutánea con repercusiones sistémicas, tales como el síndrome de Peutz-Jeghers (SPJ) e insuficiencia suprarrenal ${ }^{1}$.

El SPJ es una enfermedad hereditaria autosómica dominante de alta penetrancia, caracterizada por la presencia de poliposis gastrointestinal, aumento del riesgo de cáncer de colon y hemorragia digestiva, asociado a máculas melanocíticas en cavidad oral, bermellón, piel perioral, manos y pies. A diferencia de nuestro caso, en el SPJ existe un patrón familiar, con hiperpigmentación desde la infancia temprana. El estudio endoscópico no se considera obligatorio en ausencia de clínica sugerente. En nuestro caso, considerando el tamizaje de cáncer digestivo correspondiente a su edad, se decidió realizar el estudio y se descartó la presencia de alteraciones ${ }^{1}$.

$\mathrm{La}$ insuficiencia suprarrenal es un trastorno endocrino por déficit de producción de cortisol y aldosterona, el cual puede manifestarse con síntomas inespecíficos como dolor abdominal, fatiga o baja de peso. El aumento de ACTH produce una hiperpigmentación difusa de mucosas y zonas de presión (p. ej. nudillos y pliegues), así como aréolas mamarias y cicatrices, pudiendo ser esta la primera manifestación ${ }^{1,6,8}$. En nuestra paciente las máculas eran bien delimitadas, y se encontraba asintomática, con niveles de cortisol plasmático y ACTH dentro de rango normal.

La hiperpigmentación en el SLH no requiere tratamiento y es necesario asegurar al paciente su naturaleza benigna. En caso de preocupación estética, la criocirugía, láser N-Yag y Q-switched alexandrita son opciones seguras y eficaces ${ }^{16,17}$. La fotoprotección es recomendable para prevenir recurrencia ${ }^{4}$. Nuestra paciente no expresó deseos de tratamiento.

No existe certeza acerca de la patogénesis del SLH, pero se ha postulado que un estímulo crónico desconocido podría determinar una anormalidad en la función de los melanocitos, con aumento de la síntesis de melanosomas ${ }^{18,19}$.

Si bien la asociación entre SLH y síndrome de Sjögren podría ser fortuita, existen reportes de esta patología asociada a diversas enfermedades autoinmunes en otros países, tales como lupus eritematoso cutáneo y sistémico, artritis reuma- 
toide, síndrome de Sjögren, anemia hemolítica con Coombs positivo, médula ósea hipocelular con anticuerpos antinucleares positivos y penfigoide bulloso $^{10-14}$. La existencia de estos casos, por si solos, no son prueba suficiente para establecer una relación de causalidad, sin embargo, podrían sugerir un estímulo autoinmune para la síntesis de melanina en el SLH. Considerando lo anterior, sugerimos realizar un tamizaje clínico para estas condiciones (p. ej. fiebre, compromiso del estado general, baja de peso, artralgias, fotosensibilidad, alopecia, etc.) y solicitar anticuerpos antinucleares sólo en aquellos donde se sospeche enfermedad sistémica asociada.

Este caso es de interés para la comunidad médica debido a que sería el primer caso reportado de SLH con un estudio detallado de autoinmunidad. Se trata de una entidad altamente subdiagnosticada, con una prevalencia desconocida y probablemente subestimada ${ }^{17}$. Dado su excelente pronóstico, es necesario conocer esta patología para limitar la cantidad de estudios exhaustivos e innecesarios, disminuyendo además los costos y ansiedad del paciente. Se requiere de mayor información para determinar si el SLH es efectivamente de etiología autoinmune. Se desconoce si el hallazgo de estas patologías con SLH corresponden a hallazgos fortuitos ${ }^{12}$.

\section{Referencias}

1. Montebugnoli L, Grelli I, Cervellati F, Misciali C, Raone B. Laugier-Hunziker syndrome: An uncommon cause of oral pigmentation and a review of the literature. International Journal of Dentistry. Disponible en: http:// www.hindawi.com/journals/ijd/2010/525404/ [Consultado el 20 de julio de 2015].

2. Meng-Sui L, Hsieh-Ching C, Li-Fang W. Laugier-Hunziker syndrome. Dermatologica Sinica 2006; 24: 209-12.

3. Kanwar AJ, Kaur S, Kaur C, Thami GP. Laugier-Hunziker syndrome. J Dermatol 2001; 28: 54-8.

4. Pérez A, del Pino G, López M. Síndrome de Laugier-Hunziker. Revisión bibliográfica y presentación de 3 casos infantiles. Revista Mexicana de Cirugía Bucal y Maxilofacial 2010; 6: 14-8.

5. Asati DP, Tiwari S. Laugier-Hunziker syndrome. Indian J Dermatol Venereol Leprol 2011; 77: 536.
6. Sachdeva S, Sachdeva S, Kapoor P. Laugier-Hunziker syndrome: A rare cause of oral and acral pigmentation. J Cutan Aesthet Surg 2011; 4: 58-60.

7. Sardana K, Mishra D, Garg V. Laugier-Hunziker syndrome. Indian Pediatr 2006; 43: 998-1000.

8. Urbina F, Sudy E, Misad C, Sandoval R. Enfermedad de Laugier. Piel 1999; 13: 333-8.

9. De la Sotta P, Salomone C, González S. Síndrome de Laugier-Hunziker. Revista Chilena de Dermatología 2004; 20: 194.

10. Akcali C, Serarslan G, Atik E. The Laugier-Hunziker syndrome. East Afr Med J 2004; 8: 544-5.

11. Tan J, Greaves MW, Lee LH. Laugier-Hunziker syndrome and hypocellular marrow: A fortuitous association? Clin Exp Dermatol 2007; 32: 584-5.

12. Lalosevic J, Zivanovic D, Skiljevic D, Medenica L. Laugier-Hunziker syndrome-Case report. An Bras Dermatol 2015; 90: 223-5.

13. Kemmet D, Ellis J, Spencer MJ, Hunter JAA. The Laugier-Hunziker syndrome: a clinical review of 6 cases. Clin Exp Dermatol 1990; 15: 111-4.

14. Lamey PJ, Nolan A, Thomson E, Lewis MA, Rademaker M. Oral presentation of the Laugier-Hunziker syndrome. Br Dent J 1991; 171: 59-60.

15. Shiboski SC, Shiboski CH, Criswell L, Baer A, Challacombe S, Lanfranchi $\mathrm{H}$, et al. American college of rheumatology classification criteria for Sjögren's syndrome: a data-driven, expert consensus approach in the Sjögren's international collaborative clinical alliance cohort. Arthritis Care Res 2012; 64 (4): 475-87.

16. Ergun S, Saruhano lu A, Migliari DA, Maden I, Tanyeri H. Refractory pigmentation associated with Laugier-Hunziker syndrome following Er: YAG laser treatment. Case Rep Dent. Disponible en: http://www. hindawi.com/journals/crid/2013/561040/ [Consultado el 20 de julio de 2015].

17. Ozawa T, Fujiwara M, Harada T, Muraoka M, Ishii M. Q-switched alexandrite laser therapy for pigmentation of the lips owing to Laugier-Hunziker syndrome. Dermatol Surg 2005; 31: 709-12.

18. Mignogna M, Lo Muzio L. Laugier-Hunziker syndrome: A clinical, histopathological and ultrastructural review of 12 cases. Oral Dis 1999; 5: 80-6.

19. Veraldi S, Cavicchini S, Benelli C, Gasparini G. Laugier-Hunziker syndrome: a clinical, histopathologic, and ultrastructural study of four cases and review of the literature. J Am Acad Dermatol 1991; 25 (4): 632-6. 